\title{
Philosophiques
}

\section{La traduction française d'un ouvrage de H.G. Gadamer, Warheit und Methode}

\section{Jean-Claude Petit}

Volume 10, numéro 1, avril 1983

URI : https://id.erudit.org/iderudit/203218ar

DOI : https://doi.org/10.7202/203218ar

Aller au sommaire du numéro

Éditeur(s)

Société de philosophie du Québec

ISSN

0316-2923 (imprimé)

1492-1391 (numérique)

Découvrir la revue

Citer ce document

Petit, J.-C. (1983). La traduction française d'un ouvrage de H.G. Gadamer, Warheit und Methode. Philosophiques, 10(1), 153-175.

https://doi.org/10.7202/203218ar d'utilisation que vous pouvez consulter en ligne.

https://apropos.erudit.org/fr/usagers/politique-dutilisation/ 


\title{
LA TRADUCTION FRANÇAISE D'UN OUVRAGE DE H.G. GADAMER Warheit und Methode
}

\author{
par Jean-Claude Petit
}

La traduction française de l'ouvrage de H.G. Gadamer, Wabrheit und Methode. Grundzüge einer philosophischen Hermeneutik. J.C.B. Mohr (Paul Siebeck), Tübingen 1960 (2. Aufl. 1965, 4. Aufl. 1975) 524 p., est parue en 1976 aux Éditions du Seuil, à Paris, sous le titre: Vérité et méthode. Les grandes lignes d'une berméneutique philosophique.

Une note en exergue à la traduction se lit ainsi :

La traduction est celle de la $2^{\mathrm{e}}$ édition (1965), édition comportant quelques modifications sur le texte de 1962 (sic !) et une importante préface qu'on lira ci-dessous. Une traduction intégrale n'ayant pu être donnée, un choix, accepté par l'auteur, a été fait, qui laisse de côté la première section - de caractère historique et critique - de chacune des deux premières parties, soit : dans la première partie, la section « Die Transzendierung der ästhetischen Dimension ", p. 1-96 et, dans la deuxième partie, "Geschichtliche Vorbereitung ", p. 162-249. La traduction est d'Étienne Sacre, recteur de l'Université du SaintEsprit à Beyrouth ; elle a été possible grâce à la générosité de cette institution à l'égard du traducteur. La révision est de Paul Ricœur, qui exprime sa dette à l'égard de la traduction italienne, parue sous le titre Verità e metodo, par Gianni Vattimo, édition Fratelli-Fabbri (Milan, 1972).

L'éditeur aurait pu rappeler également que le lecteur n'y trouvera pas non plus les six Excursus sur des points particuliers qui accompagnent l'édition allemande, ni le texte "Hermeneutik und Historismus ", introduit à la fin de la seconde édition de 1965 et qui avait pour but de mieux situer les travaux 
de Gadamer dans la production contemporaine, ni non plus d'index.

Voilà déjà qui fait de cette traduction française une œuvre " inachevée ". Il est bien difficile de deviner quelles ont pu être les contraintes qui ont présidé à de telles décisions lorsqu'on connaît l'importance de l'ouvrage traduit et l'urgence qu'il y avait à le rendre accessible aux lecteurs français. Mais tout ceci aurait été simplement un moindre mal si la traduction offerte avait été de qualité. Or, tel n'est pas le cas et c'est la raison qui explique cette intervention. Traduire un texte est une entreprise difficile, voire périlleuse. Le traducteur doit sans cesse " prendre position ", prendre sur lui des décisions qui l'exposent à la critique des autres, " il ne peut rien laisser en suspens, comme le rappelle Gadamer dans l'œuvre traduite, de ce qui n'est pas clair pour lui » (WuM. p. 363), « il doit annoncer la couleur », bref il se trouve toujours sous une contrainte qui l'oblige à dire constamment, dans son travail même' de traduction, " comment il comprend ». Tâche difficile, à la vérité, tâche dangereuse. Mais " s'il prend cette tâche au sérieux " rappelle également Gadamer, le traducteur proposera un texte plus plat mais aussi « plus clair que l'original »(WuM. p. 364).

Or, il n'est pas nécessaire de lire longtemps cette traduction française de Wabrbeit und Methode pour s'apercevoir qu'elle n'est pas «plus claire que l'original ». Elle se caractérise plutôt par une quantité étonnante de fautes et de contresens ; en plus d'un endroit, le texte est inintelligible et l'accès au sens original est souvent impossible. Faute de pouvoir refaire à nouveaux frais la traduction de l'ouvrage et compte tenu de l'importance que celuici ne cesse de revêtir, il est apparu utile de proposer une liste de corrections qui rendent le texte français plus conforme à l'original. Il aurait été fastidieux de relever toutes les erreurs ou les imprécisions de la traduction. Nous avons essayé de ne retenir que les plus grossières ou celles qui risquent d'orienter le lecteur sur des pistes qui engagent dans une autre direction que celle de l'herméneutique philosophique de H.-G. Gadamer.

Parmi les concepts fondamentaux de Wabrbeit und Metbode dont l'accès est rendu difficile par la traduction en question, il y a ceux, par exemple, de «Wirkungsgeschichte » et de « wir- 
kungsgeschichtliches Bewusstsein ". En fait, ils ont été traduits de plusieurs façons en français mais sans que ces traductions n'arrivent à montrer vraiment de quoi il s'agit. Une tentative récente cependant paraît bien y être arrivée. Il s'agit de celle de $\mathrm{J}$. Grondin dans un article intitulé " La conscience du travail de l'histoire et le problème de la vérité en herméneutique ${ }^{1}$. Après E. Sacre qui propose dans sa traduction française " l'histoire de l'efficience »; P. Ricœur qui traduisait par « la conscience exposée aux effets de l'histoire " ou « la conscience de l'efficace historique »; Fataud, qui selon Ricœur proposait « la conscience insérée dans le devenir historique »; P. Fruchon, qui traduisait par " la conscience engagée dans l'histoire " et J. Greisch qui proposait «l'effectivité du sens ${ }^{2}, \mathrm{~J}$. Grondin propose de traduire la "Wirkungsgeschichte " par « le travail de l'histoire" et « wirkungsgeschichtliches Bewusstsein » par « la conscience du travail de l'histoire ». Cette traduction paraît de loin supérieure à celles qui avaient été proposées jusqu'ici et les raisons invoquées à son appui entraînent l'adhésion. On se facilitera ainsi de beaucoup la compréhension de ce qui est en question dans Vérité et méthode si on introduit à chaque fois dans le texte de la traduction « le travail de l'histoire » là où on lit « l'histoire de l'efficience ${ }^{3}$

Faculté de Théologie

Université de Montréal

1. Archives de Philosophie 44 (1981) 435-453.

2. Références dans J. Grondin, loc. cit. 436-437.

3. Ces corrections doivent beaucoup à la collaboration de $M$. le professeur Fernand Couturier, du département de philosophie de l'Université du Québec à Montréal, qui a bien voulu les relıre et nous faire part de plusieurs observations utiles. Dans la liste qui suit, le premier chiffre indique la page de la traduction française, le second renvoie à la ligne ; le chiffre entre parenthèses indique la page correspondante de l'original allemand, en l'occurrence celui de la seconde édition (1965). 
$11,34(\mathrm{XVII})$

13,5 (XVIII)

13,8 (XVIII)

$13,30(\mathrm{XVIII})$

14,13 (XIX)

$14,16(\mathrm{XIX})$

$14,40(\mathrm{XX})$

$15,1(\mathrm{XX})$

$17,30(\mathrm{XXII})$

18,1 (XXII)

18,4 (XXII)

19,7 (XXIII)

$19,10(\mathrm{XXIII})$

$19,26(X X I 11)$

19,28 (XXIV)

$19,36(\mathrm{XXIV})$

$21,2(\mathrm{XXV})$ au lieu de : «L'analyse que je consacre à l'esthétique du génie..." : "Ici, l'esthétique de génie a fourni un travail préparatoire important, dans la mesure .... ».

: lire : " tous les types d'intérêts à l'égard de l'histoire, parce qu'elle concerne à chaque fois ce qui est au fondement de la "'question de l'histoire ». »(Historische Frage)

: au lieu de «problème de l'histoire ": « question de l'histoire " (Historische Frage)

: au lieu de : "Autant dire qu'on a ... : :

"Ceci ne veut dire rien d'autre qu' . . .".

: au lieu de « vrai ": «valide».

: au lieu de : « doit être devenue problématique ": " doit avoir été mise en question".

: au lieu de : «domination par » : «détermination de ".

: au lieu de : « concerne " : « domine ».

: après «comportement subjectif », ajouter : "Le langage ne se résout pas dans la conscience de celui qui parle et est dans cette mesure davantage qu'un comportement subjectif ».

: au lieu de: «des comptes rendus " : " mes comptes rendus ».

: au lieu de : " j'ai souligné ma dette de fait ... . etc » : " que je considère effectivement la Critique de la Raison pure de Kant comme déterminante (verbindliche) et que les assertions pour qui c'est seulement sur le mode dialectique que ....".

: lire : «Heidegger aussi a dû, comme nombre de mes critiques, déplorer ici l'absence d'une dernière radicalité au moment de tirer les conséquences ".

: au lieu de: "Son achèvement dans la science?": "Que signifie son achèvement dans la science?"

: après « À.plus forte raison »: « me semblet-il».

: après " la réalité »: «dans laquelle il se trouve $"$.

: au lieu de : « ce réel " : «le réel ».

: au lieu de « outrepasse »: « dépasse ». 
$27,7(97)$

$27,13(97)$

$27,24(97)$

$28,15(98)$

$28,21(98)$

29,8 (98)

$29,26(99)$

$29,31(99)$

$29,33(99)$

$31,17(100)$

$32,18(101)$

$33,30(102)$

$34,7(103)$

$37,17(106)$

38,23 (107)

$39,26(108)$

39,27 (108)

$40,36(109)$

$41,3(109)$

41,13 (109)

41,18 (109)

41,19 (109)
: au lieu de : « ni en général la liberté ... . : « ni en aucune manière la liberté . . . ".

: lire: "Il est certain qu'on peut distinguer....".

: au lieu de : «seulement » : « tout simplement ».

: au lieu de : « donc" : " et ainsi ».

: lire : « ce qui subsiste et perdure ... . .

: au lieu de : «maintenant »: « au fond ».

: au lieu de : «Le mouvement du jeu » : «Le mouvement qui est jeu ... .».

: au lieu de : «Il ne s'agit ici que du jeu ... . " :

«C'est le jeu qui est joué ou qui se joue - il n'y a aucun sujet d'impliqué qui y joue ".

: au lieu de : " peut-être pas " : "même pas ».

: après : " aussi jouent ", lire : " et que, au sens figuré, on pourrait même dire . . . .

au lieu de: " cette possibilité n'est pas intacte »: «cette liberté n'est pas à l'abri ».

: au lieu de : « au monde des buts ordinaires »: « au monde ordinaire des buts : "

: au lieu de : « on ne peut dire " : « on peut dire ".

: au lieu de : « jeu »: « œuvre» (Werk)

: au lieu de : « ce qui doit advenir » : « qu'estce qui est visé ?"

: au lieu de : « jeu esthétique »: « jeu de l'art ».

: au lieu de: «en chaque jeu, l'être signifie... .": "l'être de tout jeu est à chaque fois libération (Einlösung) . . . .

: au lieu de : "qui cherche la vérité du langage ": "qui cherche la vérité de l'être dans les “Logoi », c'est-à-dire dans l'idéalité du langage .».

: au lieu de : « la reconnaisance » : «sa reconnaissance ».

: au lieu de : « manière ": " matière ".

: point après : essence.

: lire : "Parce qu'elles ne sont pas seulement "répétition " (Wiederholung) mais "pétition" (Hervorholung), le spectateur est en 
$42,8(110)$

$42,9(110)$

$43,26(111)$

$44,9(112)$

$44,23(112)$

$45,12(113)$

$45,17(113)$

$46,2(113)$

$46,5(113)$

$46,23(114)$

$46,27(114)$

$46,28(114)$

$48,2(115)$

$48,4(115)$

$49,12(116)$

$49,22(116)$

$49,36(117)$ même temps visé en elles. Elles comportent de soi la référence essentielle à chacun de ceux pour qui il y a représentation ».

: supprimer « par».

: au lieu de : «dans le domaine de l'art»: "pour l'esthétique".

: après " en figure" : "Le jeu est figure cette thèse veut dire : . . . .

lire : « À la double différenciation de l'œuvre littéraire et de son matériau et de l'œuvre littéraire et son exécution correspond une double non-différenciation comme l'unité de la vérité que l'on connaît dans le jeu de l'art ».

: lire : "Elle n'existe pas en soi et ne nous rencontre pas en plus par une médiation qui lui serait accidentelle, mais bien plutôt elle accède dans la médiation à son être authentique- ".

: aulieu de : «De ce fait, l'artiste qui se donne à exécuter dispose lui aussi d'une certaine initiative ": "L'interprète a lui aussi une certaine conscience de cela ".

au lieu de : "bien que ": "en cela même que ....". (indem).

: supprimer : "première ".

: au lieu de : « dans la mesure où elles .... " : " et de cette manière elles maintiennent l'identité et la continuité de l'œuvre d'art visiblement ouvertes en direction de son avenir ».

: au lieu de : « limiter. . . en recourant à » : "limiter... à ».

: au lieu de : « sans un acte créateur " : «sur un acte créateur".

: au lieu de : «par la figure » : «sur la figure ».

: lire: "cette contemporanéité et cette actualité ».

: au lieu de : « combiner " : "penser ensemble cette atemporalité et la temporalité ... .».

: au lieu de: "que la représentation peut subir ": "qu'elle peut subir dans la représentation $»$.

: au lieu de : " originale " : "originelle ".

: supprimer « or ». 
$54,5(121)$

56,24 (123)

$56,30(123)$

$57,3(123)$

$59,10(125)$

$59,11(125)$

$59,32(126)$

$61,9(127)$

$62,17(128)$

62,35 (129)

$63,10(129)$

63, 16 (129)

63,21 (129)

$64,21(130)$

$64,33(130)$

$66,25(132)$

$69,20(135)$

$69,23(135)$
: au lieu de "culturelle »: " cultuelle».

: aulieu de: «en commençant par Aristote . . . : " Si nous commençons par Aristote, nous aurons pour cette raison même une vue sur la totalité du phénomène tragique ".

: au lieu de : «la définition " : « cette définition ".

: au lieu de : « ressent » : « expérimente ».

: au lieu de: «qui représentent... . : "laquelle représente la véritable exigence démesurée pour le spectateur ».

au lieu de : " consiste à . . . " : " est la mâ̂trise de cette exigence démesurée (Zumutung) ".

au lieu de : «que goûte ": «qui goûte ». Supprimer : " volontiers ».

: au lieu de: «qui nous ont servi à caractériser »: "qui caractérisent ".

au lieu de : «s'oppose à ": «vient à la rencontre de " (va au devant de ....).

: au lieu de : «les modes d'être »: «le mode d'être ».

: au lieu de: «inspiré par »: «qui marque ....".

: au lieu de : «bien que " : «pour qui ».

: au lieu de : «création": «configuration» (Gestaltung).

: au lieu de : « univers »: «monde ". (souligné dans le texte)

au lieu de : «... demande que les représentations se dédoublent sans se distinguer ». : ". . passe par le doublement de ces représentations sans les distinguer".

: après : « . . l'image elle-même » : « dans la mesure où il s'agit précisément de la manière ... ".

: au lieu de : " parce qu'il accède à la représentation » : " parce qu'il doit représenter ».

: au lieu de : «Ce n'est pas ... . : «C'est seulement parce qu'il possède de telle manière (derart) un être dans l'acte de se montrer qu'il est proprement représenté dans l'image ». 
70,24 (136)

71,11 (136)

71,14 (136)

71,17 (136)

71,28 (137)

72,1 (137)

72,4 (137)

72,5 (137)

72,8 (137)

72,9 (137)

$75,32(140)$

$76,26(141)$

$76,42(141)$

77,6 (141)

$77,22(141)$

78,18 (142)

$78,31(143)$

$79,2(143)$

79,7 (143)

79,27 (143)

80,6 (144) après : " elles permettent ", lire : " plutôt à la chose qu'elles représentent d'être ainsi enfin pleinement ce qu'elle est ».

: supprimer «totalement».

: au lieu de : "poétique » : " plastique ».

: au lieu de: "langue ": "langage".

au lieu de "procès artistique ": « advenir de l'art» (Kunstgeschehen).

: au lieu de : «facteur »: « moment».

: "être" : Sein et non Seiendes (étant).

: au lieu de : «sur la base de " : « comme un » (als).

: au lieu de «image-tableau ": «image " (Bild).

: au lieu de "univers": «monde ".

: au lieu de : « un fait général » : « un contexte général » (Verhältnis - relation).

: au lieu de: "pureté ascétique ": "spiritualité puriste" (puristische Geistigkeit).

: au lieu de : « tout en la dépassant » : « en ce qu'il la dépasse » (indem es ihn Ubersteigt c'est en la dépassant qu'il la contient).

: au lieu de : " autrement dit » : «car ceci signifie que...."

: au lieu de "au point que ... . : lire : "quelque dégagée des circonstances que puisse être aussi l'individualité représentée dans le portrait pour accéder à l'essentiel de sa manifestation $»$.

: après solennel : « comme tel ».

: au lieu de "toujours": "toujours déjà " (immerschon).

: au lieu de «effectivité ": " actualité » (Aktualität).

: au lieu de : « un monde » : «le monde».

: lire : «me semble en constituer....».

: au lieu de: "L'œuvre d'art reçoit »: lire : "L'œuvre d'art est ainsi conçue comme un phénomène d'être et l'abstraction dans laquelle la différenciation esthétique la place est dissoute. L'image aussi est un processus de la représentation ". 
$80,35(144)$

$81,3(145)$

$82,8(146)$

$82,12(146)$

$82,13(146)$

$82,20(146)$

$82,24(146)$

$83,8(146)$

$83,29(147)$

$83,35(147)$

$84,24(148)$

$85,28(149)$

$88,22(151)$

$90,28(153)$

$91,5(153)$

$91,27(154)$

$93,21(155)$

$93,33(156)$

$94,2(156)$
: au lieu de «facteur»: « moment ».

: au lieu de "faire renvoi par soi-même ": " renvoyer loin de soi " (von sich wegzuverweisen).

: au lieu de «tout en permettant»: «en ce qu'elle permet ... (indem).

: au lieu de « elle »: " il " (das Dargestellte).

: au lieu de "l'être » : « il » (das Dargestellte).

: au lieu de : "les symboles": un «symbole" (souligné dans le texte).

: au lieu de : «celui-ci ": « le symbole».

: au lieu de " rendre présent »: « laisser être » (sein lassen).

: lire : «Il n’est pas plus là . . . " (souligné dans le texte).

: au lieu de : "Mais cela signifie qu'en elle le "modèle " - objet de la repraesentatio ... " «Mais cela signifie qu'en elle le représenté - le "modèle " - . . . "

: au lieu de: " au symbole »: « au symbole cultuel "(Kultsymbol).

: au lieu de : " La science de l'art elle-même " : "La science de l'art aussi . . . "

: au lieu de : "... ce qui est décoratif n'est pas l'œeuvre du génie... ." " ce qui est seulement décoratif n'est pas l'art du génie..."

: au lieu de : « une existence tout aussi authentique »: " une existence (Dasein) tout aussi originelle » (Ursprüngliches).

: au lieu de : "spécificité esthétique de la littérature »: "spécificité artistique de la littérature » (Kunstart).

: au lieu de : « historique ": « historienne ».

: au lieu de «qu'elles élèvent »: " qu'ils élèvent » (c.-à-d. les "langages »).

: au lieu de : « elle »: « il » (c.-à-d. le mode d'être).

: au lieu de : " quelque chuse de purement et simplement contemporain ", plutôt : " quelque chose d'absolument contemporain et familier $\gg$. 
$95,13(157)$

$97,7(159)$

$98,1(160)$

$99,12(161)$

$99,36(161)$

$103,14 / 15(250)$

$104,7-14(251)$

$104,31(251)$

$105,3(251)$

$105,10(252)$

$105,30(252)$
: au lieu de : "grâce auquel : "dans lequel " (in dem).

au lieu de : « univers " : « monde " (Welt).

lire : « À l'opposé, Hegel offre une autre possibilité d'équilibrer gains et pertes ... .»

: au lieu de : " car il est la récollection et l'intériorisation de l'esprit d'autrefois dispersé et extériorisé encore en elles " : " car il est l'intériorisation (Er-Innerung) de l'esprit encore extériorisé (ver-äusserten) en elles». (souligné dans le texte).

au lieu de : « dans la mesure où notre problème a été celui de la vérité »: " dans la mesure où nous questionnons au sujet de la vérité ».

: au lieu de : « la fondation par Heidegger de la structure circulaire de la compréhension sur la temporalité du Dasein ": " le dégagement par Heidegger de la structure circulaire de la compréhension à partir de la temporalité du Dasein ".

: Le texte cité de Heidegger doit se lire ainsi : "Le cercle ne doit pas être dégradé au rang d'un cercle vicieux, fût-il même simplement toléré. En lui se cache une possibilité positive du connaître le plus originel qui, certes, ne peut être saisie correctement que si l'interprétation a compris que sa tâche première et permanente consiste à se laisser donner à chaque fois son pré-avoir, son pré-voir et sa pré-saisie non pas par des intuitions et des notions populaires mais bien d'assurer le thème scientifique par le développement de ces anticipations à partir des choses elles-mêmes ".

: au lieu de : " a toujours un projet " : « réalise toujours un projet $»$.

: lire : « cette description» au lieu de : «la description".

: au lieu de : "entretient ": « constitue ".

: au lieu de : " de ne pas tenir comme allant de soi qu'il s'exprime . . . : « de ne pas faire intervenir sans l'avoir critiqué, son propre usage de la langue, ou, dans le cas d'une langue étrangère, l'usage qui nous est familier à partir des écrivains ou des tournures quotidiennes que nous connaissons ». 
$105,34(252)$

$107,6(253)$

$107,9(253)$

$107,33(254)$

$107,35(254)$

$108,17(254)$

$109,10(255)$

109,25 (256)

117 , note $2(262)$

$118,31(264)$

$119,5(264)$

$120,28(265)$

$120,29(265)$

$121,6(266)$

121,32 (266)

$121,36-39-41(266)$

$122,11(267)$

122,13 (267)

$123,4(268)$

$123,28(268)$
: au lieu de : " de tel usage de la langue ... . : « de l'usage de la langue du temps ou de l'auteur en question".

: lire : «La tâche berméneutique aboutit d'elle-même à une manière de questionner qui porte sur la chose et elle est toujours déjà marquée par un tel questionnement .

: au lieu de : «Pour comprendre un texte »: " celui qui veut comprendre, ne devrait pas dès l'abord s'en remettre au hasard de ses propres préconceptions....».

au lieu de : "Par là il se soumet . . . " : «au fond il n'a fait ainsi que ce que la conscience historico-herméneutique exige en chaque cas ».

: au lieu de : «méthodologique »: «méthodique $"$.

: au lieu de : " sur laquelle il appuiera son propre projet ontologique ": « à laquelle son propre projet ontologique aura à s'éprouver ».

: au lieu de : «maintenant " : « aujourd'hui ».

: au lieu de: «l'idéal méthodologique": "l'idée de méthode ".

: au lieu de : «p. $320 »:$ : p. $257 »$ (p. 110 de la traduction française).

: De : « ̀̀ mon sens . . . » jusqu'à : « n'est pas admis »: entre parenthèses dans le texte.

: au lieu de : «dans son principe " : « en principe ".

: au lieu de : «facteur» : « moment».

Supprimer: "seulement».

: au lieu de « facteur »: « moment ".

: lire: « commune au comprendre dans les sciences humaines".

: «signification»: n'est pas souligné dans le texte.

: au lieu de : «élément " : "moment».

: au lieu de : « un facteur constitutif de l'attitude historique " : « reconnaître dans l'attitude historique le moment de la tradition ».

: au lieu de : "la loi de l'objet ": « la loi de la chose en question".

: au lieu de : "Au contraire la chose ne commence... : " Au contraire, la chose en 
$123,31(268)$

$123,38(268)$

$123,40(268)$

$124,16(269)$

$125,21(270)$

$127,12(271)$

$128,2(272)$

$129,3-4(273)$

$129,24(274)$

$133,10(277)$

$133,14(277)$

$133,38(277)$

$134,17(278)$

$134,27(278)$

$135,9(279)$

$136,24-26(280)$

question ne nous apparaît vraiment signifiante que dans la lumière de celui qui sait nous la décrire comme il faut".

au lieu de : "Nous accordons que ces aspects different selon les époques et les situations historiques ": "Nous reconaissons que ce sont des aspects différents sous lesquels, à des époques et en des lieux divers, la chose en question se présente d'une manière historique ".

: au lieu de : «elle » :« il » (c.-à-d. le passé).

au lieu de: "Dans l'histoire moderne ellemême, la recherche n'est pas seulement recherche, mais aussi transmission de tradition ": " La recherche historiographique moderne ellemême n'est pas seulement recherche mais médiation de tradition ".

: lire : «Un tel objet en soi n'existe même pas ».

: au lieu de : « doit » : « devrait ».

lire : " le caractère continu de l'histoire universelle».

$\because$ au lieu de : " qui décident " : " qui décide " (c.-à-d. l'éloignement).

: au lieu de : « civilisation » : " culture».

: "parce que qu'... . : souligné dans le texte.

: lire: « la structure circulaire de la compréhension"

: au lieu de : «mouvement de va-et-vient » : "mouvement circulaire".

au lieu de : "facteur " : " moment ".

au lieu de : " naissant du rapport de l'opinion proférée à la vérité » : " qui surgissent de la relation à la vérité de ce qui est visé ".

: au lieu de : «nos propres préconceptions»: " notre propre préconception".

: après : «transmission " : « et ".

: au lieu de : «mais désigne entre l'intermédiaire et l'auteur une différence irréductible qui résulte de la distance historique qui les sépare ": "mais décrit au contraire une différence irréductible entre l'interprète et l'auteur, différence qui est donnée par et dans la distance historique ». 
$136,39(280)$

$138,29(282)$

$138,39(282)$

$139,21(283)$

$139,30(283)$

$140,2(283)$

$140,15(283)$

$140,23(283)$

$141,33(284-285)$

$141,38(285)$

$142,3(285)$

$142,21(285)$

$143,7(286)$

$143,9(286)$

$143,16(286)$

$143,28(286)$

$144,15(287)$

$144,19(287)$

$145,8(288)$
: au lieu de : «cet aveu " : « ceci ».

: lire : "Comme Aristote, qui a montré à quel affinement des possibilités humaines de juger peut conduire un tel problème, de même ici la réflexion herméneutique doit reconnaître un affinement de la conscience méthodologique de la science ".

: supprimer « en outre".

: au lieu de : " que la tradition soit isolée »: "que la tradition se démarque de son côté ".

: au lieu de : "doit déjà avoir été mis en valeur » : «doit s'être lui-même auparavant fait valoir ".

: au lieu de : «à un autre texte»: «à ce qui est autre ».

: au lieu de : "l'autre aspect de ce qui nous est propre " : « l'autre du propre » (das Andere des Eigenen).

: au lieu de : «La compréhension est par essence ... . : lire : «Comprendre est en son essence un processus historique opérant " (ou : " efficace ": ein wirkungeschichtlicher Voŕgang).

: supprimer : " pouvoir de ".

: au lieu de: "Nous n'exigeons donc pas ... . : "Il n'est donc pas exigé . . .».

: au lieu de: "L'histoire des sciences nous a appris à voir en elle . . . " : " Nous connaissons cette déformation à partir de l'histoire des sciences comme l'argumentation irréfutable en faveur de quelque chose d'évidemment faux " .

: au lieu de : «d'avoir " : «d'abord».

: au lieu de : «présence » : «actualité ».

: "Horizon» : souligné dans le texte.

: lire : «à sa détermination finie ".

: au lieu de : «horizon» : lire : «horizons».

: au lieu de : "interprète " : " celui qui veut comprendre ».

: au lieu de : "inaccessible sécurité " : "sûre inaccessibilité ».

: au lieu de : «civilisation»: «culture». 
$145,33(288)$

$146,6(288)$

$146,14-16(289)$

$147,11(289)$

$147,12-13(289)$

$147,40(290)$

$148,2 \mathrm{a}), 8(291)$

149,2 (291)

149,8 (291)

149,42 (292)

$150,7(292)$

150,24 (292)

$151,3(293)$

151,8 (293)

$151,22(293)$

$152,6(294)$

$152,16(294)$

152,25 (294)

$153,3(295)$

153, dernière ligne (296)

$154,20(296)$
: au lieu de : «son horizon » : « un horizon ».

: au lieu de : «interprète " : « celui qui veut comprendre ".

: lire : « et l'argument de Nietzsche à l'effet que l'histoire nuise à la vie n'atteint pas en réalité la conscience historique..."

: au lieu de : «qu'on prétend isoler les uns des autres ": " existant apparemment chacun pour soi ».

: au lieu de : «... dans les temps les plus anciens, dans la manière naive dont les hommes se comportent à l'égard d'eux-mêmes et de leurs origines " : « nous connaissons la force d'une telle fusion surtout à partir d'époques plus anciennes et de leur manière naïve de se situer face à elle-même et à leur origine ».

: au lieu de: "Il ne se..." : "Elle ne se ....»(c.-à-d. la projection ....).

: au lieu de : «éléments " : « moments».

: au lieu de : "éléments " : « moments ".

: au lieu de : «élément» : « moment».

au lieu de : " historique ": « historienne".

: au lieu de : «partie ": «moment ".

après : « historique ": «C'est-à-dire que là aussi ....

: au lieu de : «posée par . . . " : « posée à l'intelligence que la science moderne a d'ellemême ".

: au lieu de : « coordonner » : « justifier ».

: après : "herméneutique »: " théologique ».

: supprimer : «à l'occasion ».

après : «psychologique »: « c'est-à-dire ».

au lieu de : « ne differe pas en son principe »: " n'est, en principe, rien d'autre que . . . "

: au lieu de : «C'est au service... . : lire : «Au service de ce qui doit prévaloir, elles sont des interprétations qui incluent l'application ".

: au lieu de : «impulsion ": «tendance» (Streben).

: au lieu de : «l'agent moral »: «celui qui agit». 
$154,22(296)$

$154,26(296)$

$156,4(298)$

$157,1(298)$

$160,29(302)$

$162,5(303)$

$163,1(304)$

$163,27(305)$

$164,11(305)$

$164,29(305)$

$165,34(306)$

$166,34(307)$

$167,13(308)$

$167,31 / 32(308)$

$168,38(309)$

169 , n. 2 (309)

$171,1 \mathrm{i}(311)$

$171,22(311)$

$171,36(312)$

$172,21(312)$
: après : « savoir »: « en général ».

: "Il donne en même temps au problème de la méthode une portée morale": souligné dans le texte.

: au lieu de : " Mais c'est en tant qu'être agissant . . . " : «Il se connaît comme un être qui agit et le savoir qu'il a ainsi de lui-même ne vise pas à constater ce qui est ».

: au lieu de : "le problème ": " la conscience ".

: supprimer : " la variabilité du droit naturel ».

après : «du jugement d'équité » : «qui seul trouve vraiment ce qui est juste ».

: au lieu de : « sur le statut de ce savoir » : «sur ce à propos de quoi il vaut comme savoir ».

: au lieu de : «telles qu'on peut les rencontrer ... . : « qu'il s'agit d'atteindre ... .»

: au lieu de : « ce que cette situation a de visible en tant que telle": "ce qu'il y a de visible comme tel en cette situation".

: au lieu de : "place": «sens" (Sinn).

: au lieu de : « la vertu qu'il possède » : « la vertu qui consiste à le posséder ».

: au lieu de : "historique »: " historienne » (historisch).

: au lieu de : " et n'y avait plus vu une application": "n'y avait plus vu qu'une application ...."

: au lieu de : «historique " : historienne" (historisch).

: au lieu de : "historique ": " herméneutique ".

: supprimer : «F. Wieacker ». (cf. ligne 6).

: au lieu de: "N'est-ce pas dire qu'il exige . . . : "N'est-ce pas dire qu'une transposition est toujours nécessaire ?"

: au lieu de : «mais ": «mais au contraire" (Sondern).

: au lieu de : «qui veut comprendre " : «qui comprend ".

: au lieu de : «délibérer ": «conseiller" (beraten). 
$172,30(313)$

172,34 (313)

174,13 , etc. (314)

$175,40(315)$

176,26(316)

$178,37(318)$

$178,38(318)$

$179,9(318)$

179, $11(318)$

179, 38 (319)

$180,10(319)$

180,17 (320)

181,2 (320)

$181,20(321)$

$181,23(321)$

$181,37(321)$

$182,26(321)$

$182,40(322)$

$183,9(322)$

183,31-32 (322) au lieu de : « d'incorporer à la dogmatique juridique ": " de transformer d'une manière dogmatique » (dogmatisch zu verarbeiten).

: supprimer : « du droit».

au lieu de : "problème de Dieu " : «question de Dieu » (Gottesfrage).

: au lieu de : "interprète " : " celui qui comprend » (Verstehenden).

: au lieu de : "épistémologie ": « théorie de la science " (Wissenschaftstheorie).

: au lieu de : "pour les textes ": " pour une tradition" (solcher Überlieferung).

: au lieu de : « vient se soumettre " : « est soumis » (wird . . . . unterworfen).

: au lieu de : «de préférence » : « avant tout » (vorzüglich)

: au lieu de : «ce qui . . . : « ce que pour ainsi dire, l'expression " trahit»."

: lire : «Les textes apparaissent à côté de tout autre matériau historique, c'est-à-dire à côté des soi-disant restes" (Überreste, restants).

: au lieu de : « La tension est inévitable . . . » : " il existe une tension naturelle entre ...".

: au lieu de : «historique ": « historienne» (historische).

: au lieu de: « historique ": « historienne " (historische).

: Entre parenthèses : « sans qu'il veuille, bien entendu, en convenir ".

: au lieu de : «texte»: " quelque chose ».

: au lieu de : " si l'image qu'on a mise à la base de l'attitude historique ": "si même l'image de l'attitude historienne qui a servi de guide ici n'est pas une caricature ».

: au lieu de : «historique" : "historienne" (historisch).

: au lieu de : « historique ": « historienne" (historisch).

: lire : "pour le texte isolé comme tel ".

: au lieu de : " 11 nous semblait avoir compris ... : « Nous avions, comme nous le 
183,38 (323)

185,2 (324)

$185,6(324)$

$186,24(325)$

$186,34(325)$

$187,17(326)$

$187,18(326)$

187,28 (326)

189 , n. 1 (328)

190,21(328)

$197,23(335)$

198,29 (336)

199,8 (336)

$200,7(337)$

200,8 (337)

$200,10(337)$

$200,40(338)$

$203,12-32(340)$

203,37 (340) prétendons, mieux compris en quoi consiste lire un texte ».

: au lieu de : "présent au . . . : « dans le ».

$:$ au lieu de : " quel est ici le lien entre savoir et détermination de l'efficience ": « de quelle manière savoir et efficience s'appartiennent-ils mutuellement?"

: au lieu de : « et, dans cette mesure, de son efficience " : " et, dans cette mesure, elle agit elle-même d'une manière efficace ».

: supprimer : « tout » ainsi que la virgule après « herméneutique ".

: après : « de telle sorte que . . .» : «... dans la conscience de l'efficience, l'immédiateté et la supériorité de l'œuvre ne se dissolvent pas en un pur objet de réflexion ».

: supprimer : « aspect».

: ạu lieu de : « aspect»: « autre ».

: supprimer : « aspect».

: lire : «Ménon, d, suiv. »

: au lieu de: «Hegel prétend réaliser une médiation totale entre l'histoire et le présent »: " Hegel prétend réaliser une médiation totale de l'histoire et du présent ".

: au lieu de : «procès ": « événement "(Geschehen).

: au lieu de : «facteur » : «moment».

: au lieu de : «en-soi » lire : «l'en-soi » (das An-Sich), et au lieu de : «ce qui est « ensoi »: «ce qu'est l'en-soi ».

: «Yêtre " et "présent ": soulignés dans le texte.

: "La certitude qu'il a de lui-même" : souligné dans le texte.

: au lieu de: "doit d'abord s'instaurer ": « s'instaure d'abord".

: au lieu de : « ayant beaucoup vu et beaucoup appris ": "Parce qu'il a fait de nombreuses expériences et qu'il a appris à partir d'expériences ".

: au lieu de : « éléments » : «moments ».

: au lieu de : « aux modificatins » : « à la modification ". 
$204,12(341)$

$204,18-19(341)$

$205,10(342)$

205,36 (342)

206, 1 (342)

$206,34(343)$

$207,5(343)$

$207,20(343)$

$208,3(344)$

$210,3(346)$

$210,12(346)$

$210,21(346)$

$212,4(347)$

$212,5(347)$

$212,9(348)$
: lire : «Si nous appliquons au problème herméneutique la forme de la relation au toi et de la compréhension du toi que trahit la connaissance des hommes, ce qui lui correspond alors c'est la foi naïve en la méthode et l'objectivité que celle-ci permet d'atteindre ".

: au lieu de : "C'est en éliminant . . . " : « et en éliminant méthodiquement tous les moments subjectifs dans le rapport à la tradition, il devient certain de son contenu ".

: au lieu de : « et » : «mais».

: au lieu de : « historique ": « historienne ".

: au lieu de : « comme la compréhension du toi s'étend à l'autre » : « comme la compréhension du toi le reconnaît comme personne ».

: au lieu de : «que " : " qui ».

au lieu de: «suivre l'avis de quelqu'un»: " être à l'écoute de quelqu'un ".

: au lieu de : «la naïveté de l'assimilation » : " la manière naïve de comparer ".

: après : " nous gardons en mémoire" lire : "quelle signification revint au concept de question lors de l'analyse de la situation herméneutique ".

: au lieu de : « auxquelles on se tient " : «qui se maintiennent».

: au lieu de: "auxquelles on se tient vraiment : "qui se maintiennent vraiment ".

: au lieu de: «désigne un point indécis»: « qu'un ouvert est visé » (ein Offenes wird gemeint)

: au lieu de : « les brillantes observations aristotéliciennes ": «les brillantes observations de Ernst Kapp au sujet de la genèse de la syllogistique aristotélicienne».

: au lieu de : "Cette primauté de la question . . . : " La limite de l'idée de méthode pour le savoir, limite d'où sont parties l'ensemble de nos réflexions, se montre de la manière la plus radicale dans la primauté que possède la question pour l'essence du savoir ".

: au lieu de : "problématique » : "ce qui mérite d'être soumis à la question » (das Fragwürdige). 
$212,34(348)$

$212,38(348)$

$214,13(349)$

$215,19(350)$

$215,27(350)$

216,11(351)

$216,35(352)$

218, n. $2(353)$

$221,4,5,23(355)$

$221,16(356)$

$221,24(356)$

$222,8(356)$

$222,13-15(356)$

$223,21-22(357)$

$226,6(360)$

$230,4(361)$

232, $15(363)$

$232,19(364)$
: au lieu de : "C'est donc l'acte de questionner ": « elles présupposent donc des questions ».

: au lieu de : «Mais que la question ....»: "La venue (Einfall) de la question cependant est déjà la brèche (Einbruch) dans l'espace plat de l'opinion courante ".

: au lieu de : « en référence à ": « à partir de ».

: supprimer : « toute».

: au lieu de « verbe »: « le mot» (das Wort).

: au lieu de "procès ": « la forme d'achèvement" (Vollzugsform). Au lieu de : « verbe »: « mot ».

: au lieu de : «telle»: «tel ».

: au lieu de: «Voir sur ce point...»: «Remarques pertinentes sur ce point de.....

: au lieu de : « la voix du passé » : « la parole de la tradition " (das Wort der Überlieferung).

au lieu de : "elle-même comprise dans une interrogation plus vaste »: « elle-même posée à l'intérieur d'un questionner par et dans lequel nous cherchons....".

: au lieu de : «aller " : « être ".

: au lieu de : «de véritables questions » : « un véritable questionner ".

: au lieu de : "Il a beau rabattre . . . : «II a beau être tellement replié, à partir de la visée immédiate de la chose en question, sur la visée de sens comme telle et ne considérer celle-ci non pas comme vraie mais seulement comme pertinente (sinnvoll), que sa possibilité d'être vraie demeure en suspens, une telle mise en suspens constitue précisément ... .

: au lieu de : « ces textes»: «celle-ci » (c.-àd. la reconnaissance).

: au lieu de : «sous forme de dialogue ": « sous forme langagière ».

: au lieu de : « langue »: «langage».

: au lieu de : «ce qui lui semble obscur » : «ce qui n'est pas clair pour lui ».

: lire : « où se trouve toujours le traducteur ». 
233,32 (365)

236, 1 (367)

246,23 (376)

246,31(376)

246,35 (376)

247, 1 (376)

$247,36(377)$

$247,40(377)$

248, 12 (377)

248, 18 (377)

252,12 (381)

253, 18 (382)

254, 1 (383)

$255,14(383)$

$256,33(385)$
: au lieu de : «compliqué précisément par . . . etc. " : " c'est-à-dire un cas d'étrangeté et de son dépassement » (d. h. von Fremdheit und Überwindung derselben).

: au lieu de: «donne une dimension concrète . . . " : " est la concrétion de la conscience de l'histoire de l'efficience ".

: au lieu de : « contexte ": « texte ".

: au lieu de : « restitution " : « transmission" (Weitergabe).

: au lieu de : «principes ": « concepts".

: au lieu de : « cela signifie deux choses : d'une part . . . etc. " : "cela ne signifie pas seulement que la visée de sens du texte est accomplie d'une manière unitaire, mais que, par là, la chose dont le texte parle se porte elle-même à la parole".

: au lieu de: "l'interprétation conduite dans...": "l'interprétation exprime clairement dans le medium du langage luimême en quoi consiste . . .".

: au lieu de : " par excellence ": «point " ( überhaupt»).

: après : «précède ": "pour ainsi dire " (gleichsam).

: au lieu de : « donc » : « de la même manière » (ebenso).

: au lieu de : « un artiste qui se borne à des reproductions " : " un interprète " (ein reproduzierender Künstler).

: au lieu de : "il peut lui arriver . . . » : « il est conforme à ce qu'elle est, qu'elle disparaisse elle aussi en tant qu'interprétation . . . ».

: au lieu de : «peut »: « veut».

: au lieu de : «facteur » : « moment ».

: lire : « il ne faut pas les considérer tous les deux uniquement....».

: au lieu de : «découverte " : «econnaissance ».

: au lieu de : « n'est pas conscient du souci ": " ne connaît pas la question de ".

: au lieu de : « elle compte " : "il compte " (c.-à-d. le langage, die Sprachlichkeit). 
$257,8-9(385)$

$257,10(385)$

$258,27(386)$

$259,32(387)$

$259,32(387)$

$259,33(387)$

261, 14 (388)

264,22(391)

$267,25(394)$

$267,28(394)$

$270,29-31-35(397)$

274, n. $1(400)$

275, n. $1,2(401)$

$277,11(403)$

$285,20(410)$

286, n. $1,2(411)$

$286,24(411)$

$287,1(411)$
: lire : "Sprachgebundenheit".

: au lieu de : «facteurs » : «moments ».

: au lieu de: «Or ce qui n'est qu'un peu juste . . . " : «Il est bien possible, lorsque ce qui n'est qu'un peu juste reproduit encore en soi l'esquisse (tupos) de la chose, que cela soit suffisamment bon pour être employé ».

: au lieu de : «l'objet » : « un objet ".

: au lieu de : « désigner » : « nomme » (nennt).

: «Parce qu’il a . . . » : (c.-à-d. le mot !).

: au lieu de : « la manifestation accomplie des choses qui s'y trouvent " : "la manifestation accomplie en lui des choses » (en lui : c.-à-d. le $\log o s$ ).

: au lieu de: "connaissance »: « communication " (Mitteilung).

: au lieu de : « existant»: « existante».

: au lieu de : « dans le même mot »: «dans le mot même ".

: au lieu de : «verbe»: «parole ».

: La note devrait se lire ainsi : cf. la dissertation inédite de Christoph Wagner (Heidelberg 1957) : "Die vielen Metaphern und das eine Modell der plotinischen Metaphysik " ( Les nombreuses métaphores et l'unique modèle de la métaphysique plotinienne "), qui a porté sur les métaphores de Plotin signifiantes au plan ontologique.

: au lieu de : « renouvelé »: « répété » (wiederholt).

: au lieu de : «pense le penser " : " ou il pense la pensée "(den Gedanken).

: au lieu de : «considérée comme un tout»: « dans son entier" (insgesamt).

: au lieu de : " l'histoire de la preuve " : « l'histoire de la pensée ».

: au lieu de : « leur génération n'est pas à proprement parler . . . " : "leur génération également n'est pas en fait un événement temporel mais au contraire un mouvement de la raison ».

: au lieu de : « tout est restant " : « tout en restant $»$. 
288,27 (412)

288,30 (412)

289, 11 (413)

289,28 (414)

$291,18(415)$

$292,33(417)$

294,6 (418)

295,35 (419)

297,27 (421)

297,34 (421)

$297,38-40(421)$

304, 1 (427)

306, 17 (429)

307, 1 (429)

$308,26(431)$

309,5 (431)

316,3 (437)
: au lieu de : « aux rapports »: « au rapport» (das Verhältnis).

: au lieu de: «langue » : «langage».

: au lieu de : «connaissance »: « savoir ».

au lieu de : « chacune n'est pas exacte » : « ce n'est pas chacune qui est exacte » (nicht jeder ist genau).

au lieu de : "L'intérêt qu'il porte... . : «Son intérêt, comme celui de son époque, pour l'individualité, ne peut pas du tout être compris comme une aversion...".

: au lieu de : " la somme de tout le pensable ": " la quintessence même de tout pensable " (dem Inbegriff alles Denkbaren).

: au lieu de : « relativement autre ": « autre qui nous est pleinement relatif ».

: au lieu de : «qu'on se tienne tellement éloigné »: "qu'on soit à ce point libre à l'égard... (sich so weit freihalten).

: supprimer : «simplement».

: au lieu de : " l'être lui-même : "l'étant luimême " (Seinde).

: au lieu de : «Pour qu'une chose . . . etc. " : "Une chose qui se comporte de telle et telle façon . . ., voilà en quoi réside la reconnaissance de son altérité et de son indépendance qui présuppose chez celui qui parle, une distance propre à l'égard de la chose en question ".

: au lieu de : «que seule une science déterminée serait »: "que c'est une science déterminée qui ... . .

: au lieu de : "à partir de laquelle " : " à partir duquel ».

: au lieu de : «l'articulation » : « son articulatin».

: après : « à la totalité même de l'ordre ": note 1, cf. plus haut pp. 118 ss. (Trad. franç. pp. 50 ss.).

: au lieu de : " où bien ou " : « ou bien où ".

: au lieu de : "méthodologique ": «méthodique ". 
316,31 et ss. (438)

$316,39(438)$

$316,41(438)$

317,22 (439)

$323,4(443)$

$324,41(445)$

$326,8(446)$

$328,13(448)$

329,15 (449)

$333,36(453)$

$335,30(453)$

$347,16(465)$
: au lieu de : «l'entendre »: «l'écoute ». : après : « reconnu »: note 1 (438) : "Arist. De Sensu 473 a 3. et Met. 980 b 23-25. La priorité de l'écoute sur le voir est médiatisée par l'universalité du Logos : cette priorité-là ne contredit pas la priorité spécifique du voir sur les autres sens sur laquelle Aristote insiste (Met. A 1 et en plusieurs autres endroits) ».

: au lieu de : "linguistique »: "langagière ".

: au lieu de : « au sens »: « aux sens».

: supprimer : « comme nous voyons».

: supprimer : «ainsi ".

: supprimer : "La compréhension de ".

: au lieu de: " ne connaît que l'ouverture ... . : " connaît l'ouverture ".

: au lieu de : « qui est le sens » : « qui est sens ».

: au lieu de : « retard » : « regard ».

: lire : «.. lois mécaniques. L'ingéniosité humaine ... . .

: au lieu de : "d'enquête » : « de recherche ». 